\title{
FORUM
}

\section{A New Navigation System Using Artificial Earth Satellites}

\author{
from F. G. Smith \\ (Mullard Radio Astronomy Observatory, Cambridge University)
}

1. INTRODUCtion. In a recent letter to the Editor of The Journal of the Institution of Electrical Engineers a new radio navigational system was proposed, 1 based on the reception of a radio transmission from an artificial Earth satellite. This system has the advantages of simplicity, accuracy, and world-wide coverage, and it seems worthwhile to summarize its principles and methods of use in this Journal so that its possible applications may be more widely discussed.

2. Principle. If a radio transmission with a constant frequency is received from a satellite, the apparent frequency follows a curve like those in the diagram. The frequency variation is due to the doppler effect, which for satellites at altitudes of a few hundred miles gives a variation between I part in 40,000 above and below the true frequency as the satellite passes. The true frequency is observed at the moment when the satellite is at its point of closest approach to the observer, and the rate of change of frequency may be used as a measure of the closest distance of approach. The curves shown here, for example, were measured on the $4 \circ \mathrm{Mc} / \mathrm{s}$ transmission from Sputnik I, on transits at ranges of 350 and 135 nautical miles.
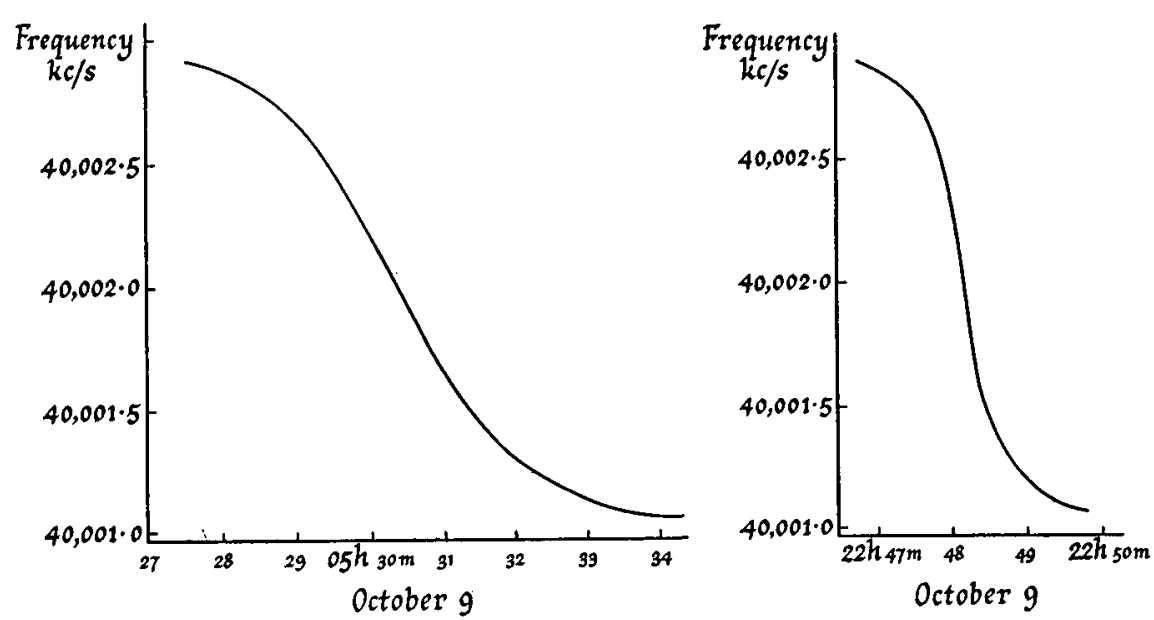

Fig. 1. The apparent frequency of the radio transmitter in Sputnik I, passing at ranges of 350 n.m. and 135 n.m.

The simplicity of the system is now evident. A single receiver, equipped only with a means of measuring frequency accurately, can be used to measure during the passage of a satellite both its minimum range and the time at which it attains 
that minimum range. If the progress of the satellite along its track is known in advance, the observer's position is at once determined.

3. Operation. Observations consist essentially of:

(i) The time of passing the true frequency.

(ii) The time taken for the frequency to sweep between two fixed frequencies on either side of the centre. These may both be achieved automatically, or by a simple presentation on a pen and ink recorder, or by a visual or audible presentation for an observer who can stand by with an eye on the clock.

4. Presentation. For a stationary observer the two measured quantities may be used directly to plot a position. A track of the satellite is required, with times marked along it. The point of closest approach may first be determined. The measured range is a slant range, and a knowledge of the satellite's height is required for this to be converted into range projected on the surface of the Earth. This projected range may be squared off the track to find the observer's position.

The procedure may be simplified by the use of a transparent chart which has a grid of times and projected ranges appropriate to a satellite with a track of given height and inclination to the ecliptic. If this chart is laid over a standard navigational chart in a relative position depending on the time and longitude of the equatorial transit of the satellite, the plotting of the results can be done directly. The transparent chart would be different for different orbits, but if the orbit were circular or if the apsides of an elliptical orbit were moving only slowly, one chart could be used for one latitude at many successive transits. Charts could, of course, be prepared some months in advance.

For a fast-moving observer the data must first be corrected for speed and heading. Without this correction the slant range could be in error by 3 per cent for a speed of 600 knots; the correction required in the apparent time of transit depends on the range as well as the speed and heading, but at this speed it would be up to about 20 miles. A simple graphical correction would be possible for this order of speed.

5. Geographical coverage. It would appear at first sight that a polar orbit would be the most desirable, since a single satellite would then be observable during two periods each day throughout the world.. Ten satellites with orbits suitably spaced in longitude would provide observations anywhere at intervals not greater than an hour and a half. However, an orbit which is inclined at $64^{\circ}$ to the equatorial plane has the great advantage that apsidal motion, which is the progressive movement of the major and minor axes of an elliptical orbit in the plane of the orbit, is zero. Since it will be very difficult to obtain a nearly circular orbit, it would be necessary to compute the apsidal motion and to make frequent corrections to the charts for the change in height of the satellite unless the $64^{\circ}$ orbit were chosen.

The coverage from such an orbit would depend on the height of the satellite, but at heights of about 400 nautical miles the only part of the globe not covered would be within $5^{\circ}$ of the poles.

6. ACcuracY. The accuracy will be limited by uncertainties in the orbit, by irregularities in radio propagation, and by the precision with which measurements can be made. The orbit of a large and easily visible object can be determined very accurately, and at 400 miles height it should be possible even without further 
research on air density and the figure of the Earth to predict the position of the orbit within a mile for a few days in advance. Beyond this time the variations in air friction, which are at present not understood, would reduce the accuracy. The position of the satellite in its orbit is less easily determined in advance, since variations in air friction have a greater effect: it might be necessary to distribute information about the time of crossing the equator no more than one day in advance to obtain a comparable accuracy. Over a whole week errors of up to ten miles might accumulate, although we may expect present experiments to show this to be a very pessimistic estimate.

Irregularities in propagation through the ionosphere and atmosphere have been demonstrated in the $40 \mathrm{Mc} / \mathrm{s}$ signals from Sputniks I and III. For this reason and from consideration of power consumption it would appear that a frequency in the region of 200 to $300 \mathrm{Mc} / \mathrm{s}$ would be a better choice, and here the irregularities would give negligible errors. The ionospheric disturbances which disrupt communications at lower frequencies would have no effect at all. The signal would, however, still fade periodically during a transit, owing to the Faraday rotation of polarization in the ionosphere.

Accuracy of measurement depends on the signal-to-noise ratio at the receiver. To allow for the fading of the signal several observations of the falling apparent frequency will be necessary, using a series of narrow frequency filters on the receiver. Several pairs of frequencies will then be available, and the centre time should be obtainable to about 0.2 sec., corresponding to I mile in position. Slant range should be obtainable to about $\frac{1}{4}$ per cent; the error in position here depends on the apparent elevation of the satellite at transit. If the satellite is at $45^{\circ}$ elevation the accuracy will be about $1 \frac{1}{2}$ miles; if it is directly overhead the position is unknown to about $3 \circ$ miles, and a good position must wait for observations of the next transit $1 \frac{1}{2}$ hours later.

7. ConClusion. It appears that a simple system of navigation could be set up giving world-wide coverage with an accuracy of about one mile. If the system were widely used, the cost of the satellites and of a central computing service would be relatively low; the receivers are little more than crystal controlled communications receivers. The presentation is simple, and can be done by graphical methods.

The main disadvantage of the system is the limitation in its usefulness to the times at which a satellite may be observed over the horizon. Even with ten satellites the gap between observations may be $\mathrm{I} \frac{1}{2}$ hours, and it would be hard to reduce this interval. At first sight it would seem that the system could be most useful for shipping, where errors in dead reckoning over such an interval are small. It is possible, however, that long-distance aircraft could use it with advantage.

REFEREN CE

1 Ryle, M. and Smith, F. G. (1959). J. Instn elect. Engrs, 5, 168.

\section{Navigation in Channel Swimming}

\section{from Commander Gerald Forsberg, O.B.E., R.N.}

ON 26 August 1875 , Captain Matthew Webb landed in France after swimming for 21 hours, 45 minutes. This master mariner (and former Conway boy) was 